\title{
Toll-Like Receptor 2 Gene Polymorphisms Associated with Aggressive Periodontitis in Japanese
}

\author{
Marika Takahashi ${ }^{1,2}$, Zhiyong Chen ${ }^{2,3,5}$, Kaoru Watanabe ${ }^{1}$, Hiroaki Kobayashi ${ }^{1}$, Toshiaki \\ Nakajima $^{2,4}$, Akinori Kimura ${ }^{2,4} *$ and Yuichi Izumi ${ }^{1}$
}

${ }^{1}$ Section of Periodontology, Department of Hard Tissue Engineering, Graduate School of Medical and Dental Science, ${ }^{2}$ Department of Molecular Pathogenesis, Medical Research Institute, ${ }^{3}$ Department of Vascular and Applied Surgery, Graduate School of Medical and Dental Sciences, and ${ }^{4}$ Laboratory of Genome Diversity, Graduate School of Biomedical Science, Tokyo Medical and Dental University, ${ }^{5}$ Department of Rheumatology and Immunology, The Affiliated Drum Tower Hospital, Nanjing University Medical School, Nanjing, China

\begin{abstract}
Background and Objective: Aggressive periodontitis is a rare and very severe periodontal disease of early onset, which is closely associated with Porphyromonas.gingivalis (P.g.) infection in the Japanese population. TLR2 encodes Toll-like receptor 2 , which plays an important role in the protective response to $P$. $g$. infection. We investigated a possible association between TLR2 and aggressive periodontitis.

Material and Methods: Of 2,460 Japanese patients with periodontitis, 38 patients with aggressive periodontitis were enrolled in this study. These 38 aggressive periodontitis patients and 190 Japanese healthy controls were examined for an insertion/deletion (Ins/Del) polymorphism in exon 1, a polymorphism in intron 1 (rs7696323), and a synonymous polymorphism in exon 3 (rs3804100) in TLR2.

Results: We found significant associations of resistance to aggressive periodontitis with the Ins allele (allele frequency in the patients versus controls, 0.540 vs. 0.676 , OR $=0.56,95 \%$ confidence interval $(\mathrm{CI}) ; 0.34-0.92, \mathrm{p}=0.022)$ and the $\mathrm{T}$ allele of rs3804100 ( 0.579 vs. $0.716, \mathrm{OR}=0.55,95 \% \mathrm{CI}$; 0.33-0.91, $\mathrm{p}=0.018$ ), although the $\mathrm{C}$ allele of rs 7696323 showed no significant association $(0.733$ vs. 0.829 , OR=0.58). A permutation test of Ins/Del-rs7696323-rs3804100 haplotype revealed a significant association between Ins-C-T haplotype (0.252 vs. $0.479, \mathrm{p}=0.0003)$ and resistance to aggressive periodontitis.

Conclusions: The TLR2 polymorphisms were suggested to confer protection against aggressive periodontitis in a Japanese population. The association should be replicated in other cohorts to further identify the responsible TLR polymorphism(s) involved in the pathogenesis of aggressive periodontitis.
\end{abstract}

Keywords: TLR2, polymorphism, aggressive periodontitis, haplotype.

\section{INTRODUCTION}

Aggressive periodontitis $(\mathrm{AgP})$ is a rare and severe phenotype of periodontitis characterized by rapid bone destruction and attachment loss in the absence of any systemic disease, often with familial aggregation, according to the 1999 International Classification of Periodontal Diseases criteria. The rate of AgP crisis is almost $0.03 \%$ in Japanese. Periodontitis is caused by infection with gram-negative bacteria and the subsequent inflammatory response induces destruction of periodontal tissue. Progression of $\mathrm{AgP}$ is so rapid that, if not treated, supporting tissues around teeth may be lost early in the life. If predisposing factors to $\mathrm{AgP}$ would be defined, patients carrying the predisposing factors could be treated in an early stage to prevent disease progression

*Address correspondence to this author at the Department of Molecular Pathogenesis, Medical Research Institute, Tokyo Medical and Dental University, 1-5-45 Yushima, Bunkyo-ku, Tokyo 113-8510, Japan;

Tel: +81-3-5803-4905; Fax: +81-3-5803-4907, Email: akitis@mri.tmd.ac.jp and to save their teeth. It is well known that susceptibility to periodontitis is influenced by both environmental (such as older age, smoking status, plaque control) and genetic factors. To reveal the genetic predisposing factors to periodontal diseases, many association studies have targeted functional polymorphisms in genes associated with inflammatory cytokines for chronic periodontitis. For example, Kornman firstly reported in 1997 that chronic periodontitis was related to interleukin (IL)-1 (IL-1) polymorphism [1] . Following this report, many other studies attempted to elucidate the associations of chronic periodontitis (CP) with polymorphisms in other genes, including $I L-6$ and Vitamin D receptor $(V D R)[2,3]$. These results suggested that unregulated production of inflammatory cytokines would be a risk factor for chronic periodontitis.

Association studies for AgP have also been performed for inflammatory cytokine genes, reporting that the susceptibility to AgP was associated with a $I L-13$ polymorphism at 1112 in Taiwanese [4], and IL-1 $\alpha(I L-1 A)$ polymorphism at +4845 and IL-1 $\beta$ (IL-1B) polymorphism at -511 were asso- 
ciated in Chinese males [5]. In addition, the association of $\mathrm{AgP}$ with the $I L-1$ gene cluster region was investigated in an Italian population, which demonstrated a significant association with polymorphisms in the genes for IL-1 receptor antagonist $(I L 1-R N)$ and in $I L-1 B$ [6]. Furthermore, other gene polymorphisms in genes for oestrogen receptor- $\alpha$ (ERA), $V D R$, N-formylpeptide receptor $(F P R), \mathrm{CC}$ chemokine receptor 2 (CCR2), and monocyte chemoattractant protein I $(M C P-1)$ were reported to be associated with $\mathrm{AgP}$ in some ethnic groups [7].

Porphyromonas gingivalis (P.g.) is a predominant gramnegative periodontopathic bacterium which is strongly associated with $\mathrm{AgP}$ in Japanese populations $[8,9]$, although Aggregatibacter actinomycetemcomitans (A.a.) is the strongest candidate of $\mathrm{AgP}$ causative bacterium in other ethnic populations $[10,11]$. Component of P.g. is reported to be a ligand for TLR2, although A.a. is recognized by TLR4 [12], suggesting that TLR2 plays a key role in protective response against P.g. in Japanese AgP. However, there have been few studies on the relationship between $\mathrm{AgP}$ and polymorphisms, in the TLR genes. Although one study demonstrated a significant association between $\mathrm{CP}$ and a polymorphism in TLR4 [13], another study could not replicate the significant association [3]. On the other hand, no association was observed with a TLR2 polymorphism, Agg753Gln, in a German population [14], and a study in Japanese subjects also showed no association of CP with the TLR2 polymorphisms, Arg677Trp and Arg753Gln [13]. TLRs are type I integral membrane glycoproteins, which play crucial roles in the innate immune system via recognizing molecules derived from pathogens. There are different TLR types, and different TLRs recognize a variety of pathogen-associated molecules, including lipids and nucleic acids [15]. It was reported that TLR2 is involved in the recognition of pathogenic bacteria in periodontal disease [16-18]. Although the association of $\mathrm{AgP}$ with TLR2 polymorphisms accompanied by amino-acid replacement was not significant, another functional polymorphism, an Ins/Del in exon 1, which is related to the expressivity of TLR2 [19], was not tested for the association. In this study, we investigated three tag polymorphisms of TLR2 including the Ins/Del polymorphism in association with $\mathrm{AgP}$ in Japanese. We report here the association of TLR2 with protection against $\mathrm{AgP}$.

\section{MATERIALS AND METHODS}

\section{Subjects}

Two thousands and four hundreds sixty patients with periodontitis who visited Tokyo Medical and Dental University Dental Hospital from June 2009 to July 2010, were examined. All AgP cases, a total of 38 unrelated Japanese patients (21 males and 17 females; diagnosed with $\mathrm{AgP}$ based on the 1999 International Workshop for Classification of Periodontal Diseases and Conditions), were recruited for the study. Age at diagnosis of the patients was $34.4 \pm 6.1$ years. Written informed consent was given by each patient before blood sampling. Genomic DNAs from blood were prepared by a standard method. DNA samples from 190 Japanese individuals with no systemic disease were prepared from healthydonor derived B cell lines deposited to the Health Science Research Resource Bank, Japan Health Sciences Foundation
Table 1. Clinical Characteristics of AgP Patients

\begin{tabular}{|c|c|}
\hline Clinical Parameters $^{\# 1}$ & \\
\hline Smokers (\%) & 58.1 \\
\hline Number of teeth present (including wisdom teeth) & $26.5 \pm 3.5$ \\
\hline Mean probing pocket depth (mm) & $3.8 \pm 1.0$ \\
\hline Frequency of bleeding on probing (\%) & $57.9 \pm 28.7$ \\
\hline Frequency of alveolar bone loss (\%) & $41.0 \pm 23.0$ \\
\hline Rate of probing pocket depth >4 $\mathrm{mm}(\%)$ & $76.0 \pm 22.6$ \\
\hline Rate of probing pocket depth $>7 \mathrm{~mm}(\%)$ & $37.0 \pm 22.3$ \\
\hline
\end{tabular}

${ }^{\# 1}$ Value was represented as mean \pm standard deviation, when it was appropriate.

(http://www.jhsf.or.jp/English/index_e.html). Periodontal conditions in these 190 healthy controls were unknown, although inclusion of possible $\mathrm{AgP}$ patients in the control would decrease the power to detect the association. The research was conducted in full accordance with ethical principles, including the World Medical Association Declaration of Helsinki (version 2002), and the study protocol was approved by the Ethics Review Committee of the Faculty of Dentistry, Tokyo Medical and Dental University, and that of the Medical Research Institute, Tokyo Medical and Dental University.

\section{Clinical Information}

Periodontal parameters and radiographs of all $\mathrm{AgP}$ patients were assessed at their first visit. Probing pocket depth (PPD) was measured at 6 sites per tooth. Bleeding on probing (BOP) was also recorded at 6 sites per tooth. Alveolar bone loss (BL) was investigated at two sites per tooth on the radiographs. The data for frequency of BOP, rate of PPD over $4 \mathrm{~mm}$, rate of PPD over $7 \mathrm{~mm}$, and frequency of alveolar bone loss are summarized in Table $\mathbf{1}$.

\section{Genotyping of TLR2}

Human TLR2 is located on chromosome $4 \mathrm{q} 32$ and is composed of three exons. Two tag single nucleotide polymorphism (SNP), rs7696323 in intron 1 and rs3804100 in exon 3, were selected from the International HapMap project database (HapMap Data Phase III/Rel\#2, Feb09, on NCBI B36 assembly, dbSNP b126) by cut-off criteria of minor allele frequency $>0.1$ and $r$ square $<0.8$ in the Japanese population. In addition, an insertion/deletion (Ins/Del) polymorphism at -196 to -174 was investigated. The restriction fragment length polymorphism method was used for the genotyping of rs7696323 and rs3804100. Briefly, polymerase chain reaction (PCR) was performed with primer pairs, 5'ggccctagctcctgtcc-3' and 5'-gtgtagcccetgcttctcc-3' for rs7696323 typing and 5'-tcatttggcatcattggaaa-3' and 5'gagttgcggcaaattcaaag-3' for rs3804100 typing. The PCR condition was $95^{\circ} \mathrm{C} 5$ min followed by 35 cycles of $95^{\circ} \mathrm{C} 30$ sec, $58^{\circ} \mathrm{C}$ or $53^{\circ} \mathrm{C} 30 \mathrm{sec}$ for rs 7696323 or rs 3804100 , respectively, and $72^{\circ} \mathrm{C} 30 \mathrm{sec}$, and a final extension step of $72^{\circ} \mathrm{C} 10 \mathrm{~min}$. The PCR products were digested by BsrI and MwoI for rs7696323 and rs3804100 typing, respectively, followed by electrophoresis in 3\% agarose gel. The Ins/Del typing was done by PCR with a primer pair, 5'gcggactttcccttttgct-3' and 5' -gtgcagagagaccacacgag-3' under 


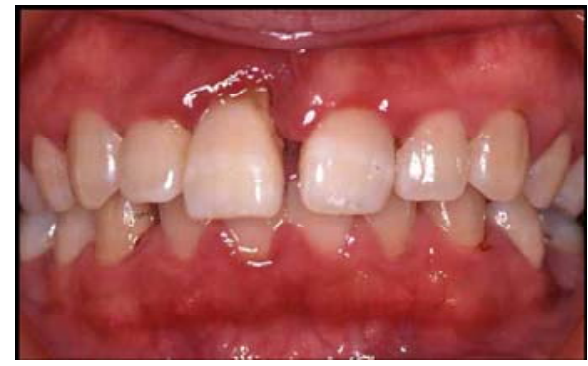

Fig. (1). A photograph of teeth from a typical AgP case. The patient was 23 years old. She was severely affected with swollen gum and almost losing the upper right incisor tooth.

the PCR condition described above, except that the annealing temperature was $57^{\circ} \mathrm{C}$ and electrophoresis was done in $2 \%$ agarose gel.

\section{Statistical Analysis}

Allele and genotype frequencies of the polymorphisms in the patients and controls were obtained by direct counting. Departure from Hardy-Weinberg equilibrium (HWE) was calculated by chi-square test. Statistical significance of differences between the AgP patients and control subjects were assessed by Mantel-Haenzel chi-square analysis, which was combined with the $2 \times 2$ contingency tables using the GraphPad in Stat software (GraphPad Software Inc., La
Jolla, CA, USA). Fisher's exact test was used if the number of any cells of the 2 x 2 contingency table was $<5$. Relative risk with $95 \%$ confidence interval (CI) was calculated as the odds ratio. Linkage disequilibrium (LD) among the alleles of tested markers and their haplotype frequencies was estimated by using the Haploview program (Haploview 4.2, http://www.broad.mit.edu/mpg/haploview/index.php). Permutation test $(n=5,000)$ was also performed by using Haploview. $\mathrm{P}$ values $<0.05$ were considered to be statistically significant.

\section{RESULTS}

Clinical parameters of enrolled AgP patients were shown in Table 1. Every clinical data showed severe progression of periodontal disease at their ages. A typical AgP case is shown in Fig. (1). The patient was 23 years old female, who showed gum of swelling and redness, and her upper right incisor tooth almost came out. She had serious problems in her mouth, but had no systemic disease other than the severe periodontitis. The allele and genotype frequencies of tested markers are listed in Table $\mathbf{2}$. It was found that the allele frequencies of Ins/Del alleles and T/C alleles of rs3804100 showed statistically significant associations with $\mathrm{AgP}$, with $\mathrm{p}$ values 0.0022 and 0.0018 , respectively, although these associations were not statistically significant after correction for multiple testing (Bonferroni's correction). Because there

Table 2. Case-Control Association Study of TLR2 Polymorphisms in Japanese AgP

\begin{tabular}{|c|c|c|c|c|c|c|}
\hline \multicolumn{2}{|c|}{ Genetic Marker } & \multirow[t]{2}{*}{$\begin{array}{l}\text { Patient } \\
(\mathrm{n}=38)\end{array}$} & \multirow[t]{2}{*}{$\begin{array}{c}\text { Control } \\
(\mathbf{n}=190)\end{array}$} & \multirow[t]{2}{*}{$\mathbf{O R} \mathbf{R}^{\# 4}$} & \multirow[t]{2}{*}{$95 \% \mathrm{CI}^{\# 5}$} & \multirow[t]{2}{*}{$\mathbf{p}$} \\
\hline-196 to -174 Ins > Del & & & & & & \\
\hline \multirow[t]{4}{*}{ genotype frequency $^{\# 1}$} & Ins/Ins & $12(31.6 \%)$ & $87(45.8 \%)$ & & & \\
\hline & Ins/Del & $17(44.7 \%)$ & $83(43.7 \%)$ & & & \\
\hline & Del/Del & $9(23.7 \%)$ & $20(10.5 \%)$ & 2.64 & $1.09-6.36$ & 0.026 \\
\hline & $\mathrm{HWE}^{\# 3}$ & $\mathrm{p}=0.83$ & $\mathrm{p}=1.00$ & & & \\
\hline \multirow[t]{2}{*}{ Allele frequency ${ }^{\# 2}$} & Ins & $41(53.9 \%)$ & $257(67.6 \%)$ & 0.56 & $0.34-0.92$ & \multirow{2}{*}{0.022} \\
\hline & Del & $35(46.1 \%)$ & $123(32.4 \%)$ & 1.78 & $1.08-2.94$ & \\
\hline \multicolumn{7}{|l|}{ rs7696323 C>T } \\
\hline \multirow[t]{4}{*}{ genotype frequency } & $\mathrm{C} / \mathrm{C}$ & $20(52.6 \%)$ & $133(70.0 \%)$ & 0.48 & $0.23-0.97$ & 0.038 \\
\hline & $\mathrm{C} / \mathrm{T}$ & $16(42.1 \%)$ & $49(25.8 \%)$ & & & \\
\hline & $\mathrm{T} / \mathrm{T}$ & $2(5.3 \%)$ & $8(4.2 \%)$ & & & \\
\hline & HWE & $\mathrm{p}=0.87$ & $\mathrm{p}=0.46$ & & & \\
\hline \multirow[t]{2}{*}{ Allele frequency } & $\mathrm{C}$ & $56(73.7 \%)$ & $315(82.9 \%)$ & 0.58 & & \\
\hline & $\mathrm{T}$ & $20(26.3 \%)$ & $65(17.1 \%)$ & 1.73 & & \\
\hline \multicolumn{7}{|l|}{ rs3804100 T>C } \\
\hline \multirow[t]{4}{*}{ genotype frequency } & $\mathrm{T} / \mathrm{T}$ & $12(31.6 \%)$ & $97(51.1 \%)$ & 0.44 & $0.21-0.93$ & 0.028 \\
\hline & $\mathrm{T} / \mathrm{C}$ & $20(52.6 \%)$ & $78(41.1 \%)$ & & & \\
\hline & $\mathrm{C} / \mathrm{C}$ & $6(15.8 \%)$ & $15(7.9 \%)$ & & & \\
\hline & HWE & $\mathrm{p}=0.89$ & $\mathrm{p}=0.99$ & & & \\
\hline \multirow[t]{2}{*}{ Allele frequency } & $\mathrm{T}$ & $44(57.9 \%)$ & $272(71.6 \%)$ & 0.55 & $0.33-0.91$ & \multirow{2}{*}{0.018} \\
\hline & $\mathrm{C}$ & $32(42.1 \%)$ & $108(28.4 \%)$ & 1.83 & $1.10-3.04$ & \\
\hline
\end{tabular}

${ }^{\# 1, ~ \# 2}$ Summation of genotype or allele frequencies at each locus may not be 1.00 because each frequency was rounded.

${ }^{\# 3}$ HWE; Hardy-Weinberg equilibrium.

${ }^{\# 4}$ Odds ratio

${ }^{\# 5} 95 \%$ confidence interval was calculated when $\mathrm{p}$ value was less than 0.05 . 
Table 3. Permutation Test for Difference in TLR2 Haplotype Frequencies in Ag Patients and Controls

\begin{tabular}{|c|c|c|c|c|c|}
\hline Haplotype $^{\# 1}$ & $\begin{array}{c}\text { Frequency in Patients } \\
(\mathbf{n}=\mathbf{3 8})\end{array}$ & $\begin{array}{c}\text { Frequency in Controls } \\
(\mathbf{n}=\mathbf{1 9 0})\end{array}$ & OR $^{\# 2}$ & $\mathbf{p}$ & $\mathbf{p c}^{\# 4}$ \\
\hline \hline Del-C-C & 0.393 & 0.253 & 1.90 & 0.013 & $\mathrm{~ns}$ \\
\hline Ins-T-T & 0.259 & 0.167 & 1.75 & $\mathrm{~ns}$ & $\mathrm{~ns}$ \\
\hline Ins-C-T & 0.252 & 0.479 & 0.37 & 0.0003 & 0.0015 \\
\hline Del-C-T & 0.068 & 0.070 & 0.97 & $\mathrm{~ns}$ & $\mathrm{~ns}$ \\
\hline Ins-C-C & 0.024 & 0.027 & 0.89 & $\mathrm{~ns}$ \\
\hline
\end{tabular}

${ }^{\# 1}$ Alleles at Ins/Del-rs7696323-rs3804100 composing of the TLR2 haplotype.

${ }^{\# 2}$ Odds ratio.

${ }^{\# 3}$ Not significant.

${ }^{\# 4} \mathrm{P}$ value was corrected for multiple comparison in haplotype frequency $(\mathrm{n}=5)$.

were only two alleles at each locus, it was difficult to determine which was responsible for the association (for example, whether the Ins allele was protective or the Del allele was susceptible). On the other hand, significant deviations in the genotype distribution were found for Del/Del $(\mathrm{OR}=2.64$, $\mathrm{p}=0.026), \mathrm{C} / \mathrm{C}$ at rs7696323 (OR=0.48, $\mathrm{p}=0.038)$, and $\mathrm{T} / \mathrm{T}$ at rs3804100 $(\mathrm{OR}=0.44, \mathrm{p}=0.028)$. These data implied that both resistance and susceptibility to $\mathrm{AgP}$ might be controlled by $T L R 2$ locus.

Because these three polymorphic markers were selected on the basis that they were not in strong LD, we estimated the frequencies of Ins/Del- rs7696323-rs3804100 haplotypes in the patients and controls using Haploview and investigated the association with $\mathrm{AgP}$ by the permutation test. The results showed that Ins-C-T haplotype and Del-C-C haplotype were significantly associated with the protection against $(\mathrm{p}=0.0003)$ and susceptibility to $(\mathrm{p}=0.013) \mathrm{AgP}$, respectively. When we assume multiple testing for the permutation test, $p$ values should be multiplied by the number of presumed haplotypes $(n=5)$. As a result, the association with Del-C-C haplotype did not show statistical significance (Table 3). Therefore, the comprehensive association studies at the allele and genotype level and at the haplotype level suggested a significant association of TLR2 locus with protection against $\mathrm{AgP}$ in Japanese.

\section{DISCUSSION}

$\mathrm{AgP}$ is a rare serious disease causing tooth loss at a young age. A characteristic of $\mathrm{AgP}$ is that it is not secondary to systemic disease accompanied by gum disease. In this study, all $38 \mathrm{AgP}$ patients have no systemic disease, and healthy-donor was selected as the control group. Another characteristic of $\mathrm{AgP}$ is the rapid progression of periodontal disease. It has been considered that $\mathrm{AgP}$ is caused by the abnormal immune response to periodontopathic bacteria, resulting in the failure to eliminate P.g. bacteria, in the Japanese cases. As $P$. $g$. is recognized by TLR2, TLR2 might play an important role in protective response against $P . g$. infection in AgP. Because TLR2 is polymorphic, some investigators have investigated TLR2 polymorphisms in association with $\mathrm{CP}$ and/or AgP, but they could not identify any significant associations [13, 14]. However, previous studies had focused on SNPs with amino acid replacement and several other TLR2 polymorphisms were not tested in the previous studies. These observations prompted us to investigate the association of $\mathrm{AgP}$ with $T L R 2$ polymorphisms so far not tested, including the Ins/Del polymorphism in exon 1 which regulates the gene expression [20].

In this study, we revealed for the first time two TLR2 polymorphisms, Ins/Del and rs3804100, which were significantly associated with AgP. A cross-sectional case-control study of polymorphic markers cannot distinguish whether one allele of each polymorphic locus is associated with the susceptibility or whether the other allele of the same locus is associated with the protection or resistance to the disease. To distinguish these possibilities, a prospective cohort study is required. However, a haplotype association study of several polymorphic alleles which were not in strong LD may be useful to evaluate the contribution of markers or their combination in association with the disease. In our study, from the investigation of each polymorphic marker, we can only conclude that TLR2 polymorphism was associated with the susceptibility or resistance to AgP. However, the permutation test for the association with presumed haplotypes revealed that the protection against $\mathrm{AgP}$ was associated with a specific TLR2 haplotype, Ins-C-T. The analysis also suggested that none of the associated marker alleles were responsible for the protection, because the frequency of Ins-T$\mathrm{T}$ haplotype was increased in the patients and the frequency of Ins-C-C haplotype was virtually identical in the patients and controls. Therefore, the protection against $\mathrm{AgP}$ was assumed to be controlled by an unidentified polymorphism in close LD to the Ins-C-T haplotype. Genetic information on the TLR2 locus might be useful in diagnosis, prevention and treatment of $\mathrm{AgP}$. If the genetic information would be obtained in young age, it might allow preventive strategies and therapeutic intervention for high-susceptibility patients.

In conclusion, we found a significant association of TLR2 polymorphisms and $\mathrm{AgP}$ in Japanese. The haplotype analysis suggested that a specific TLR2 haplotype, not the analysed SNPs, conferred protection against AgP. Because this is the first report of an $\mathrm{AgP}$-associated TLR2 haplotype, the association should be validated in other cohorts. Finally, detailed analysis of variations in TLR2 gene including rare alleles is required to further clarify the responsible SNP(s) for protection against AgP. Growing knowledge about the genetic factors that predispose individuals to $\mathrm{AgP}$ will hopefully open 
the novel insights in developing for prediction and prevention of the disease in high-risk individuals.

\section{CONFLICT OF INTEREST}

None declared.

\section{ACKNOWLEDGMENTS}

This study was supported in part by Grant-in-Aids from the Ministry of Education, Science, Sports, Culture and Technology (MEXT) of Japan, a grant for Japan-Korea collaboration research program from Japan Society for the Promotion of Science (JSPS), and research grants from The Institute of Seizon and Life Sciences. This work was also supported in part by the follow-up grants provided from the Tokyo Medical and Dental University. All authors have no conflicts of interests.

\section{REFERENCES}

[1] Kornman KS, Crane A, Wang HY, et al. The interleukin-1 genotype as a severity factor in adult periodontal disease. J Clin Periodontol 1997; 24: 72-7.

[2] Brett PM, Zygogianni P, Griffiths GS, et al. Functional gene polymorphisms in aggressive and chronic periodontitis. J Dent Res 2005; 84: 1149-53.

[3] Kobayashi T, Nagata T, Murakami S, et al. Genetic risk factors for periodontitis in a Japanese population. J Dent Res 2009; 88: 113741.

[4] Wu YM, Chuang HL, Ho YP, Ho KY, Tsai CC. Investigation of interleukin-13 gene polymorphisms in individuals with chronic and generalized aggressive periodontitis in a Taiwanese (Chinese) population. J Periodontal Res 2010; 45: 695-701.

[5] Li QY, Zhao HS, Meng HX, et al. Association analysis between interleukin-1 family polymorphisms and generalized aggressive periodontitis in a Chinese population. J Periodontol 2004; 75: 1627-35.

[6] Scapoli C, Trombelli L, Mamolini E, Collins A. Linkage disequilibrium analysis of case-control data: an application to generalized aggressive periodontitis. Genes Immun 2005; 6: 44-52.

[7] Meng $\mathrm{H}$, Xu L, Li Q, Han J, Zhao Y. Determinants of host susceptibility in aggressive periodontitis. Periodontol 2000-2007; 43: 133-59.

[8] Yano-Higuchi K, Takamatsu N, He T, Umeda M, Ishikawa I. Prevalence of bacteroides forsythus, Porphyromonas gingivalis and
Actinobacillus actinomycetemcomitans in subgingival microflora of Japanese patients with adult and rapidly progressive periodontitis. J Clin Periodontol 2000; 27: 597-602.

Miura M, Hamachi T, Fujise O, Maeda K. The prevalence and pathogenic differences of Porphyromonas gingivalis fimbriae: a genotypes in patients with aggressive periodontitis. J Periodontal Res 2005; 40: 147-52.

[10] Zambon JJ. Actinobacillus actinomycetemcomitans in human periodontal disease. J Clin Periodontol 1985; 12: 1-20.

[11] Haubek D, Ennibi OK, Poulsen K, Vaeth M, Poulsen S, Kilian M. Risk of aggressive periodontitis in adolescent carriers of the JP2 clone of Aggregatibacter (Actinobacillus) actinomycetemcomitans in Morocco: a prospective longitudinal cohort study. Lancet 2008; 371: $237-42$

[12] Sun Y, Shu R, Li CL, Zhang MZ. Gram-negative periodontal bacteria induce the activation of toll-like receptors 2 and 4, and cytokine production in human periodontal ligament cells. J Periodontol 2010; 81: 1488-96.

[13] Fukusaki T, Ohara N, Hara Y, Yoshimura A, Yoshiura K. Evidence for association between a toll-like receptor 4 gene polymorphism and moderate/severe periodontitis in the Japanese population. J Periodontal Res 2007; 42: 541-5.

[14] Schroder NW, Meister D, Wolff V, et al. Chronic periodontal disease is associated with single-nucleotide polymorphisms of the human TLR-4 gene. Genes Immun 2005; 6: 448-51.

[15] Akira S, Uematsu S, Takeuchi O. Pathogen recognition and innate immunity. Cell 2006; 124: 783-801.

[16] Schroder NW, Opitz B, Lamping $\mathrm{N}$, et al. Involvement of lipopolysaccharide binding protein, CD14, and toll-like receptors in the initiation of innate immune responses by treponema glycolipids. J Immunol 2000; 165: 2683-93

[17] Harokopakis E, Albzreh MH, Martin MH, Hajishengallis G. TLR2 transmodulates monocyte adhesion and transmigration via Rac1and PI3K-mediated inside-out signaling in response to Porphyromonas gingivalis fimbriae. J Immunol 2006; 176: 7645 56.

[18] Burns E, Bachrach G, Shapira L, Nussbaum G. Cutting Edge: TLR2 is required for the innate response to Porphyromonas gingivalis: activation leads to bacterial persistence and TLR2 deficiency attenuates induced alveolar bone resorption. J Immunol 2006; 177: 8296-300.

[19] Tahara T, Arisawa T, Wang F, et al. Toll-like receptor 2 -196 to 174del polymorphism influences the susceptibility of Japanese people to gastric cancer. Cancer Sci 2007; 98: 1790-4.

[20] Noguchi E, Nishimura F, Fukai H, et al. An association study of asthma and total serum immunoglobin $\mathrm{E}$ levels for toll-like receptor polymorphisms in a Japanese population. Clin Exp Allergy 2004; 34: 177-83.

Received: August 30, $2011 \quad$ Revised: November 10, 2011

(C) Takahashi et al.; Licensee Bentham Open.

This is an open access article licensed under the terms of the Creative Commons Attribution Non-Commercial License (http://creativecommons.org/licenses/by-nc/3.0/) which permits unrestricted, non-commercial use, distribution and reproduction in any medium, provided the work is properly cited. 